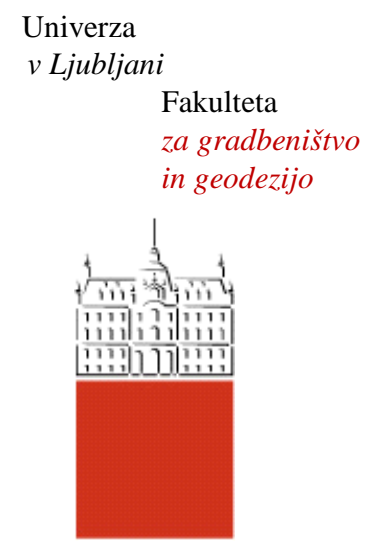

Jamova 2

1000 Ljubljana, Slovenija http://www3.fgg.uni-lj.si//

\section{DRUGG - Digitalni repozitorij UL FGG http://drugg.fgg.uni-lj.si/}

Ta članek je avtorjeva zadnja recenzirana različica, kot je bila sprejeta po opravljeni recenziji.

Prosimo, da se pri navajanju sklicujte na bibliografske podatke, kot je navedeno:
University
of Ljubljana

Faculty of

Civil and Geodetic

Engineering

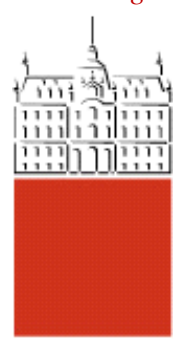

Jamova 2

SI - 1000 Ljubljana, Slovenia

http://www3.fgg.uni-lj.si/

DRUGG - The Digital Repository http://drugg.fgg.uni-lj.si/

This version of the article is author's manuscript as accepted for publishing after the review process.

When citing, please refer to the publisher's bibliographic information as follows:

Shifrin, E. I., Brank, B. 2000. On solution of elliptical interface crack problem. In: Ramm, A. G. (ed.), Shivakumar, P. N. (ed.), Strauss, A. V. (ed.). Operator theory and its applications, (Fields Institute Communication Series, vol. 25). Providence, American Mathematical Society: p. 485-496. 


\title{
On Solution of Elliptical Interface Crack Problem
}

\author{
Efim I. Shifrin \\ Department of Mathematics \\ Moscow State Aviation Technology University \\ Orshanskaya str. 3, Moscow 121552, Russia. \\ efim@shifrin.msk.ru \\ Boštjan Brank \\ Slovenian National Building and Civil Engineering Institute \\ Dimičeva 12, 1000 Ljubljana, Slovenia and \\ Faculty of Civil and Geodetic Engineering \\ University of Ljubljana, Jamova 2, 1000 Ljubljana, Slovenia. \\ bostjan . brankozag.si
}

\begin{abstract}
The problem of crack of elliptical shape located between two bonded dissimilar elastic half spaces and subjected to arbitrary loads is considered. A solution is obtained by using corresponding traction boundary pseudodifferential equations. Resulting equations are then solved by means of an analytical-numerical method. Strain energy release rates along the crack contours are presented for elliptical cracks with different ratios of semiaxes, and for half spaces with different ratios of elastic constants. For special cases the results are compared with the available exact solutions.
\end{abstract}

\section{Introduction}

Solutions of the three-dimensional interface crack problems play an important role in the strength evaluation of composite structures. However, up to now only few such problems have been solved.

Available analytical solutions are restricted exclusively to the penny-shaped crack. In particular, the problem of the penny-shaped crack subjected to normal uniform load was considered in $[\mathbf{4}]$ and $[\mathbf{3}]$. An analytical expression for the strain energy was obtained in [4], and the stress intensity factors and the strain energy release rates were calculated in $[\mathbf{3}]$. The shear load case was solved in [11], where an analytical expression for the strain energy was derived. Recently, the problem of interface penny-shaped crack subjected to uniform normal and shear loads was reconsidered in [6]; however, no numerical results concerning stress intensity factors or strain energy release rates were given. 
Solutions of interface crack problems posess a square root and oscillating singularity near the crack contour. It is extremely difficult to extract this kind of singularity by means of numerical calculations. This is the reason why there are no accurate numerical solutions in the vicinity of a crack front for the three-dimensional interface crack problems. At the same time, the asymptotic of the solution near the crack contour is its most important characteristic, since it defines the possibility of the crack growth. Therefore, obtaining new accurate solutions for the three-dimensional interface cracks is of present research interest.

An analytical-numerical method for solving elliptical interface crack problem was developed by the authors in [7]. Examples considered in [7] were restricted to the uniform normal loading conditions. The objective of this work is to apply the method developed in $|7|$ to the elliptical interface cracks subjected to the uniform as well as to the non-uniform shear loads.

\section{Analytical-numerical method}

In this section we briefly recall the analytical-numerical method developed in [7].

Let a region $G$ in the plane $x_{3}=0$ of an infinite elastic solid be occupied by a flat crack. Material of the half space $x_{3}>0$ is defined by a shear modulus $\mu_{1}$ and Poisson's ratio $\nu_{1}$, and of the half space $x_{3}<0$ by $\mu_{2}$ and $\nu_{2}$, respectively. Suppose, that the loads applied to the crack surfaces are equal in magnitude and opposite in directions:

$$
\pm t(x)= \pm\left(t_{1}(x), t_{2}(x), t_{3}(x)\right)
$$

where $x=\left(x_{1}, x_{2}\right)$.

As it was shown in [11], the problem can be reduced to the following traction boundary pseudodifferential equations with respect to the crack opening displacements $[u(x)]=\left(\left[u_{1}(x)\right],\left[u_{2}(x)\right],\left[u_{3}(x)\right]\right)$ :

$$
P_{G} A(D)[u(x)]=t(x), \quad x \in G \quad \text { and } \quad[u(x)]=0, \quad x \notin G .
$$

$P_{G}$ is a restriction operator to the crack area $G$, and $A(D)$ is a matrix pseudodifferential operator with the symbol

$$
A(\xi)=\frac{|\xi|}{g^{2}-d^{2}}\left(\begin{array}{ccc}
g-e \eta_{2}^{2} & e \eta_{1} \eta_{2} & d i \eta_{1} \\
e \eta_{1} \eta_{2} & g-e \eta_{1}^{2} & d i \eta_{2} \\
-d i \eta_{1} & -d i \eta_{2} & g
\end{array}\right)
$$

where

$$
\xi=\left(\xi_{1}, \xi_{2}\right), \quad|\xi|=\sqrt{\xi_{1}^{2}+\xi_{2}^{2}}, \quad \eta_{j}=\frac{\xi_{j}}{|\xi|}, j=1,2,
$$

and constants $g, d$ and $e$ are expressed by the shear moduli and Poisson's ratios as

$$
\begin{gathered}
g=\frac{1-\nu_{1}}{\mu_{1}}+\frac{1-\nu_{2}}{\mu_{2}}, \quad d=\frac{1}{2}\left(\frac{1-2 \nu_{1}}{\mu_{1}}-\frac{1-2 \nu_{2}}{\mu_{2}}\right), \\
e=\frac{g c+d^{2}}{g+c}, \quad c=\frac{\nu_{1}}{\mu_{1}}+\frac{\nu_{2}}{\mu_{2}} .
\end{gathered}
$$

Recall, that the pseudodifferential operator acts on vector-functions $[u(x)]$ as

$$
A(D)[u(x)]=F^{-1}(A(\xi)[\widetilde{u}(\xi)]),
$$


where $[\tilde{u}(\xi)]=\int_{R^{2}}[u(x)] e^{i(x, \xi)} d x$ is the Fourier transform, $(x, \xi)=x_{1} \xi_{1}+x_{2} \xi_{2}$, and $F^{-1}(\widetilde{g}(\xi))=\frac{1}{(2 \pi)^{2}} \int_{R^{2}} \tilde{g}(\xi) e^{-\imath(x, \xi)} \mathrm{d} \xi$ is the inverse Fourier transform.

Suppose, that $G$ is an elliptical region

$$
G=E l a=\left\{x: \frac{x_{1}^{2}}{a_{1}^{2}}+\frac{x_{2}^{2}}{a_{2}^{2}} \leq 1\right\},
$$

and assume, without loss of generality, that $a_{2} \leq a_{1}$.

Suppose also, that the applied loads may be represented by the infinite power series

$$
t_{k}(x)=\sum_{i, j=0}^{\infty} A_{i j}^{k} x_{1}^{\imath} x_{2}^{j}=\sum_{i . j=0}^{\infty} A_{i, j}^{* k} y_{1}^{2} y_{2}^{3}, \quad k=1,2,3,
$$

where $A_{\imath j}^{* k}$ are constants and $y_{j}$ are defined as

$$
y_{j}=\frac{x_{3}}{a_{j}}, j=1,2 .
$$

It is now possible to look for the solution of (2.1) in the following form:

$$
\left[u_{k}(x)\right]=\sum_{p, q=0}^{\infty} B_{p q}^{k} T_{p q}^{1 / 2}(x), \quad k=1,2,3,
$$

where $B_{p q}^{k}$ are constants, and functions $T_{p q}^{1 / 2}(x)$ are defined as

$$
T_{p q}^{1 / 2}(x)=y_{1}^{p} y_{2}^{q} \varphi_{a}^{1 / 2}(x), \quad \text { where } \quad \varphi_{a}^{1 / 2}(x)=\sqrt{1-y_{1}^{2}-y_{2}^{2}}
$$

As shown in [7], substitution of (2.2) and (2.3) into (2.1) leads to the system of equations of the form

$$
\begin{aligned}
& \frac{1}{g^{2}-d^{2}} \quad\left\{g \sum_{p, q=0}^{\infty} B_{p q}^{1} U^{p q}(x)+e \sum_{p, q=0}^{\infty} B_{p q}^{1} R_{22}^{p q}(x)-\right. \\
&\left.e \sum_{p, q=0}^{\infty} B_{p q}^{2} R_{12}^{p q}(x)-d \sum_{p, q=0}^{\infty} B_{p q}^{3} L_{1}^{p q}(x)\right\}=\sum_{, j=0}^{\infty} A_{\imath j}^{* 1} y_{1}^{2} y_{2}^{3}, \\
& \frac{1}{g^{2}-d^{2}} \quad\left\{-e \sum_{p, q=0}^{\infty} B_{p q}^{1} R_{12}^{p q}(x)+g \sum_{p, q=0}^{\infty} B_{p q}^{2} U^{p q}(x)+\right. \\
& \frac{1}{g^{2}-d^{2}} \quad\left\{d \sum_{p, q=0}^{\infty} B_{p q}^{2} B_{p q}^{1} L_{11}^{p q}(x)+d \sum_{p, q=0}^{\infty} B_{p q}^{2} L_{2}^{p q}(x)+d \sum_{p, q=0}^{\infty} B_{p q}^{3} L_{2}^{p q}(x)\right\}=\sum_{i, j=0}^{\infty} A_{i j}^{* 2} y_{1}^{i} y_{2}^{3}, \\
&\left.g \sum_{p, q=0}^{\infty} B_{p q}^{3} U^{p q}(x)\right\}=\sum_{i, j=0}^{\infty} A_{i j}^{* 3} y_{1}^{3} y_{2}^{j} .
\end{aligned}
$$

Functions $U^{p \eta}(x)$ and $R_{23}^{r^{\prime \prime q}}(x)$ are polynomials of order $p+q$. Explicit expressions for $U^{p q}(x)$ and $R_{i, 3}^{p q}(x)$ were obtained in [1] and [2] (they are also provided in [7]). Functions $L_{1}^{p q}(x)$ and $L_{2}^{p q}(x)$ may be decomposed into an infinite power series as shown in $[7]$.

Equations (2.4) represent equalities of infinite power series. By equating coefficients on the left hand side with the corresponding coefficients on the right hand 
side, one obtains an infinite system of linear algebraic equations with the constants $B_{p q}^{k}$ as unknowns. To solve this infinite system of linear equations numerically, it has to be truncated and reduced to the finite system $p+q \leq N, \quad i+j \leq N$.

Once the constants $B_{p q}^{k}$ are obtained, it is possible to calculate the strain energy release rates along the crack contour as described below (see [7] for details).

On the elliptical crack contour, which may be written in a parametric form as

$$
x_{1}=a_{1} \cos \theta, \quad x_{2}=a_{2} \sin \theta,
$$

we define functions $\left[u_{k}^{O}(x)\right]$ as

$$
\left[u_{k}^{0}(x)\right]=\sum_{p, q=0}^{\infty} B_{p q}^{k} \cos ^{p} \theta \sin ^{q} \theta, \quad k=1,2,3 .
$$

The stress iutensity factor $K_{I I I}$ at a point $x=\left(a_{1} \cos \theta, a_{2} \sin \theta\right)$ may be then expressed as (see $\mid \mathbf{7}]$ )

$$
K_{I H}=\frac{\mu_{*}}{2} \sqrt{\frac{\pi}{a_{1} a_{2}}}\left\{\frac{-\left[u_{1}^{0}(x)\right] a_{1} \sin \theta+\left[u_{2}^{0}(x)\right] a_{2} \cos \theta}{\left(a_{1}^{2} \sin ^{2} \theta+a_{2}^{2} \cos ^{2} \theta\right)^{1 / 4}}\right\},
$$

where

$$
\mu_{*}=\frac{2 \mu_{1} \mu_{2}}{\mu_{1}+\mu_{2}} .
$$

Let us further define a function $\left[u_{n}^{0}(x)\right]$ and a constaut $\varepsilon$ as

$$
\begin{gathered}
{\left[u_{n}^{0}(x)\right]=\frac{\left[u_{1}^{0}(x)\right] \frac{a_{2} \cos \theta+\left[u_{2}^{0}(x)\right] a_{1} \sin \theta}{\left(a_{1}^{2} \sin ^{2} \theta+a_{2}^{2} \cos ^{2} \theta\right)^{1 / 2}},}{\varepsilon=\frac{1}{2 \pi} \ln \frac{\mu_{2} \kappa_{1}+\mu_{1}}{\mu_{1} \kappa_{2}+\mu_{2}}, \quad \kappa_{\jmath}=3-4 \nu_{j}, \quad j=1,2,}}
\end{gathered}
$$

respectively. As it is shown in [7], the values of $K_{I}^{2}+K_{I I}^{2}$ (where $K_{I}$ and $K_{I I}$ are stress intensity factors at a point $x$ ) can be then written in the following form

$$
\begin{aligned}
K_{I}^{2} & +K_{I I}^{2} \\
= & \frac{\left(1+4 \varepsilon^{2}\right) \cosh ^{2}(\pi \varepsilon)}{g^{2}} \frac{\pi}{a_{1} a_{2}}\left(a_{1}^{2} \sin ^{2} \theta+a_{2}^{2} \cos ^{2} \theta\right)^{1 / 2}\left\{\left[u_{3}^{0}(x)\right]^{2}+\left[u_{n}^{0}(x)\right]^{2}\right\} .
\end{aligned}
$$

Finally, by using $(2.5),(2.6)$ and the formula for the strain energy release rate $J$ (see e.g. [5], $[\mathbf{9}]$ ), we can calculate the value of $J$ at a point $x$ of the crack contour as

$$
J=\frac{g}{4 \cosh ^{2}(\pi \varepsilon)}\left(K_{I}^{2}+K_{I I}^{2}\right)+\frac{1}{2 \mu_{*}} K_{I I I}^{2}
$$

\section{Numerical examples and verification of results}

3.1 Shear load in the direction of larger semiaxis. Let us begin with a uniform shear load of the following type:

$$
t(x)=(\tau, 0,0) .
$$

Results of the strain energy release rate calculations are presented in dimensionless form

$$
J_{*}(\varphi)=\frac{J(\varphi) \mu_{1}}{\tau^{2} a_{2}}
$$




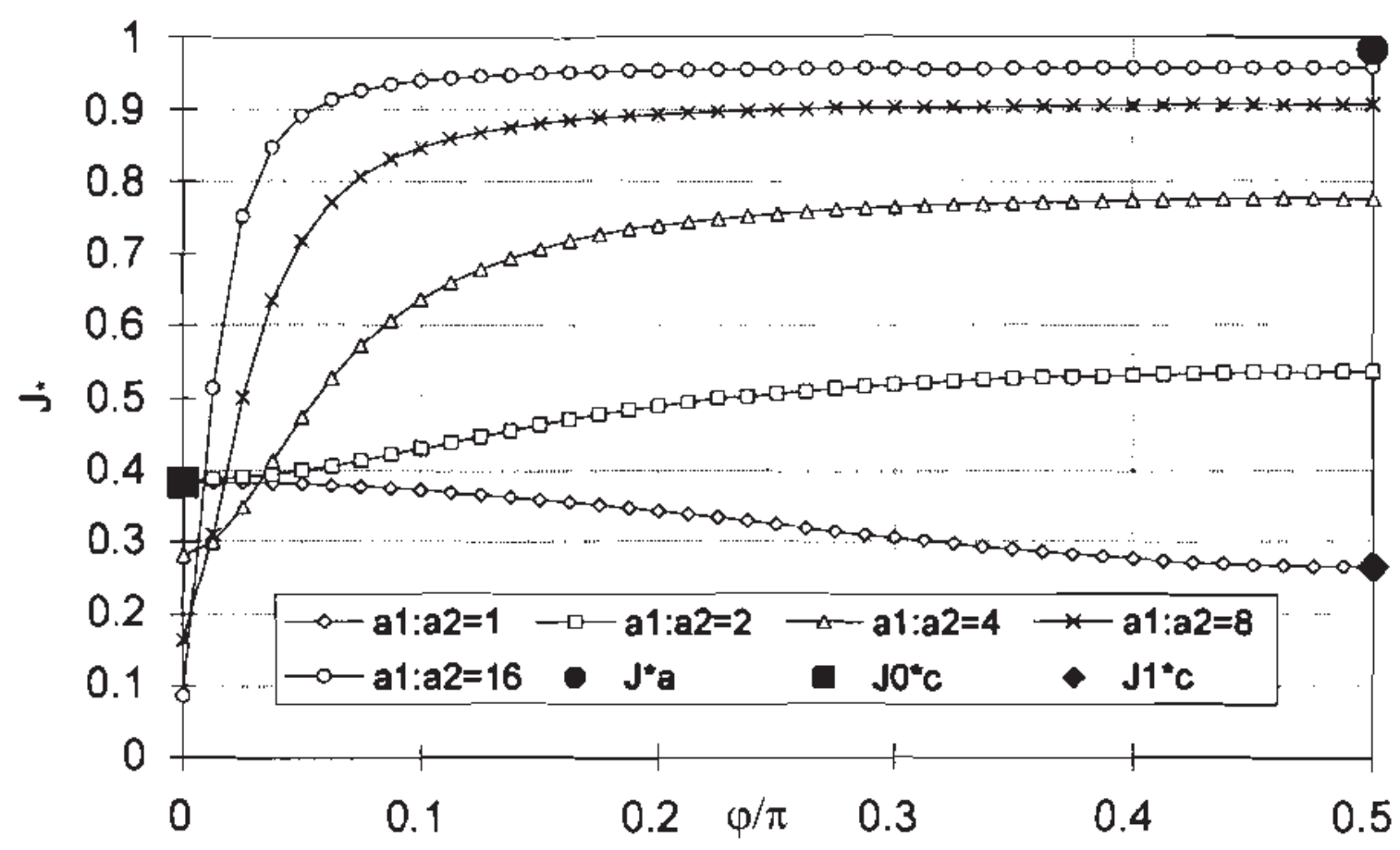

Figure 1 Shear load in the direction of larger semiaxis. Dimensionless strain energy release rates for $\nu_{1}=\nu_{2}=0.3, \mu_{1}: \mu_{2}=1: 4$.

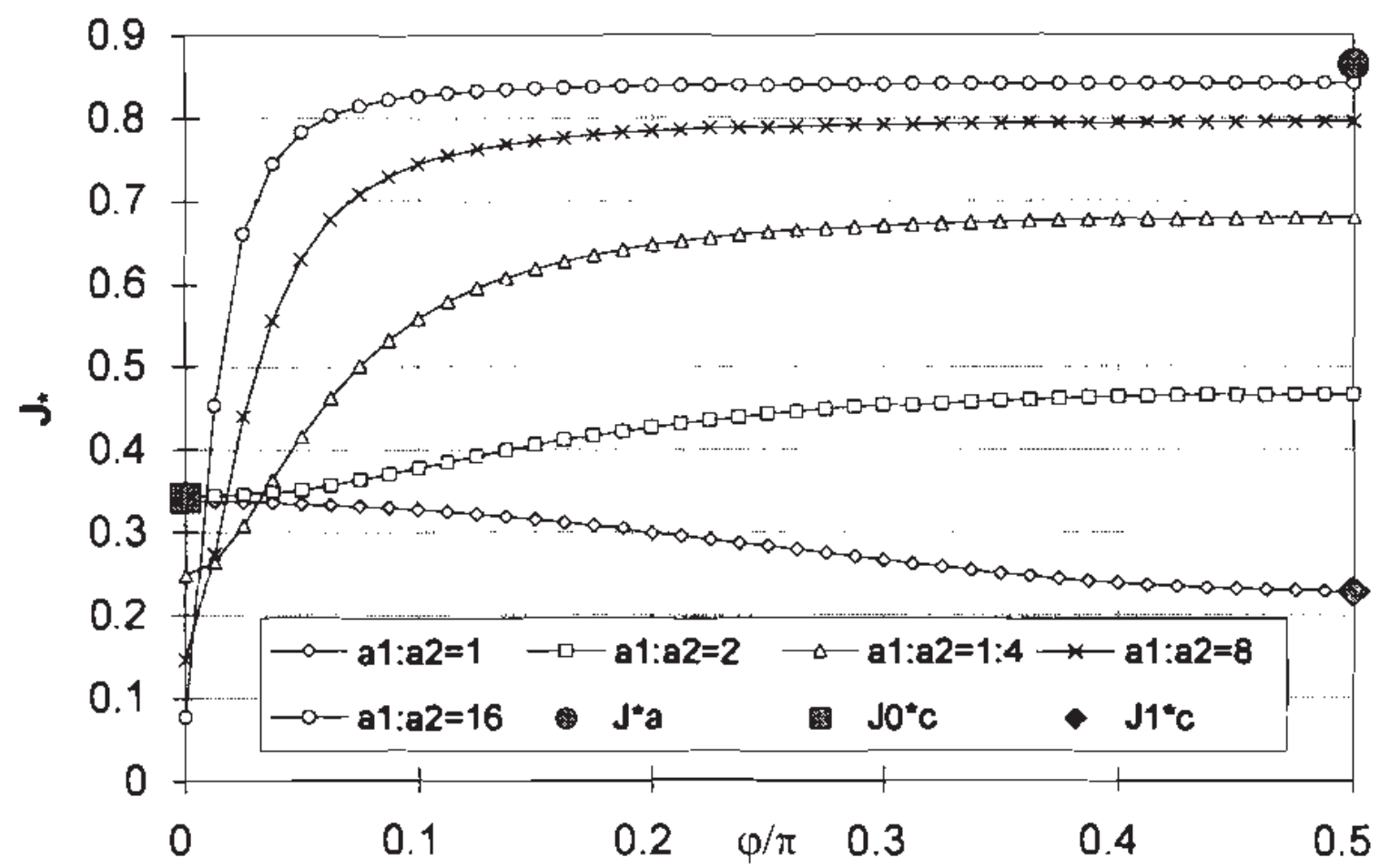

Figure 2 Shear load in the direction of larger semiaxis. Dimensionless strain energy release rates for $\nu_{1}=\nu_{2}=0.3, \mu_{1}: \mu_{2}=1: 10$.

where $\varphi$ is a polar angle in the plane $\left(x_{1}, x_{2}\right)$

$$
\varphi=\arctan \frac{x_{2}}{x_{1}}=\arctan \left(\frac{a_{2}}{a_{1}} \tan \theta\right) .
$$

As mentioned above, in the numerical procedure the infinite system of linear algebraic equations is truncated and reduced to the finite system of equations with $p+q \leq N$ and $i+j \leq N$. In order to illustrate stabilization of results with increase of $N$, a dependence of numerical values $J_{*}(\varphi)$ on $N$ is given in Table 1. Results are presented at three points $(\varphi=0, \varphi=\pi / 4$ and $\varphi=\pi / 2)$ for a particular case of a 


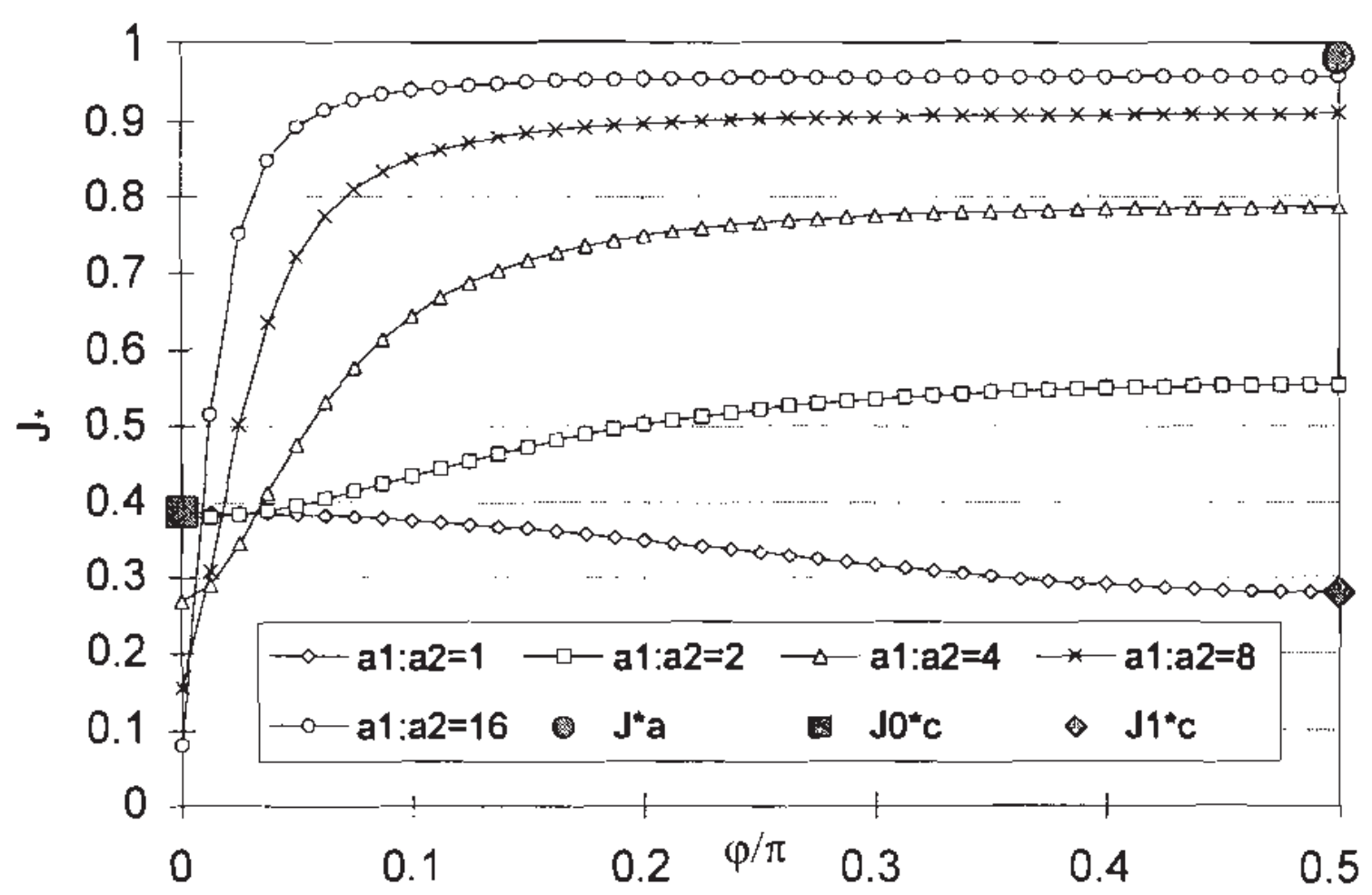

Figure 3 Shear load in the direction of larger semiaxis. Dimensionless strain energy release rates for $\nu_{1}=0.2, \nu_{2}=0.4, \mu_{1}: \mu_{2}=1: 4$.

penny-shaped crack of radius $a$. Poisson's ratio of both materials is $\nu_{1}=\nu_{2}=0.3$ and the shear moduli ratio is $\mu_{1}: \mu_{2}=1: 4$.

Convergence character of $J_{*}(\varphi)$ presented in Table 1 is analogous to the one obtained for the case of normal load (see [7]). Convergence of the results for other types of shear loads, for different nonhomogeneous solids with various material constants, and for elliptical cracks with different semiaxes ratios are similar to the one presented in Table 1.

Graphs of functions $J_{*}(\varphi)$ for three types of nonhomogeneous solids and for elliptical cracks with the semiaxes ratios $a_{1}: a_{2}=1,2,4,8,16$ are presented on Figures $1-3$ on an interval $0 \leq \varphi \leq \pi / 2$.

For elongated elliptical cracks, the value $J_{*}(\pi / 2)$ have to converge to the value of $J_{* a}$, which is a dimensionless strain energy release rate for the interface crack of antiplane shear with the length $2 a_{2}$. According to $[\mathbf{1 0}]$ and $[\mathbf{8}]$

$$
J_{* a}=\frac{\pi \mu_{1}}{2 \mu_{*}} .
$$

It can be seen from Figures 1-3, that $J_{*}(\pi / 2)$ for elliptical crack with $a_{1}: a_{2}=16$ is only slightly lower then $J_{* a}$.

For a penny-shaped crack the results obtained by analytical-numerical method may be verified as follows. According to $[\mathbf{1 1}], J_{*}(\varphi)$ fill the interval

$$
J_{* c}=\frac{4 \varepsilon\left(1+\varepsilon^{2}\right)\left(g^{2}-d^{2}\right) \mu_{1}}{\left\{(g-e) \pi \varepsilon\left(1+\varepsilon^{2}\right)+d\right\}^{2}}\left[d+\left(\pi \varepsilon\left(1+\varepsilon^{2}\right) \frac{g^{2}-d^{2}}{g+c}-d\right) \lambda^{2}\right],
$$

where $0 \leq \lambda \leq 1$. It follows from (3.2), that the extreme values of $J_{* c}$ occur when $\lambda=0$ and when $\lambda=1$. These values are equal to

$$
J_{* c}^{o}=\frac{4 \varepsilon\left(1+\varepsilon^{2}\right)\left(g^{2}-d^{2}\right) d \mu_{1}}{\left\{(g-e) \pi \varepsilon\left(1+\varepsilon^{2}\right)+d\right\}^{2}}
$$


Table 1 Dependence of $J_{*}$ on the number of equations $\mathrm{N}$ for the uniform shcar load acting in the direction of axis $r_{1}$. Penny-shaped crack; $\iota_{1}=\nu_{2}=$ $0.3, \mu_{1}: \mu_{2}=1: 4$.

\begin{tabular}{|c||c|c|c||c||c|c|c|}
\hline$N$ & $J_{*}(0)$ & $J_{*}(\pi / 4)$ & $J_{*}(\pi / 2)$ & $N$ & $J_{*}(0)$ & $J_{*}(\pi / 4)$ & $J_{*}(\pi / 2)$ \\
\hline \hline 0 & 0.3766 & 0.3186 & 0.2605 & 11 & 0.3842 & 0.3244 & 0.2646 \\
\hline 1 & 0.3859 & 0.3257 & 0.2655 & 12 & 0.3820 & 0.3232 & 0.2644 \\
\hline 2 & 0.3798 & 0.3217 & 0.2635 & 13 & 0.3841 & 0.3243 & 0.2646 \\
\hline 3 & 0.3851 & 0.3250 & 0.2650 & 14 & 0.3821 & 0.3233 & 0.2644 \\
\hline 4 & 0.3808 & 0.3224 & 0.2640 & 15 & 0.3840 & 0.3243 & 0.2646 \\
\hline 5 & 0.3847 & 0.3247 & 0.2648 & 16 & 0.3822 & 0.3234 & 0.2645 \\
\hline 6 & 0.3813 & 0.3228 & 0.2642 & 17 & 0.3839 & 0.3243 & 0.2646 \\
\hline 7 & 0.3845 & 0.3246 & 0.2647 & 18 & 0.3823 & 0.3234 & 0.2645 \\
\hline 8 & 0.3816 & 0.3230 & 0.2643 & 19 & 0.3839 & 0.3242 & 0.2646 \\
\hline 9 & 0.3843 & 0.3245 & 0.2646 & 20 & 0.3824 & 0.3234 & 0.2645 \\
\hline 10 & 0.3819 & 0.3231 & 0.2644 & 21 & 0.3838 & 0.3242 & 0.2646 \\
\hline
\end{tabular}

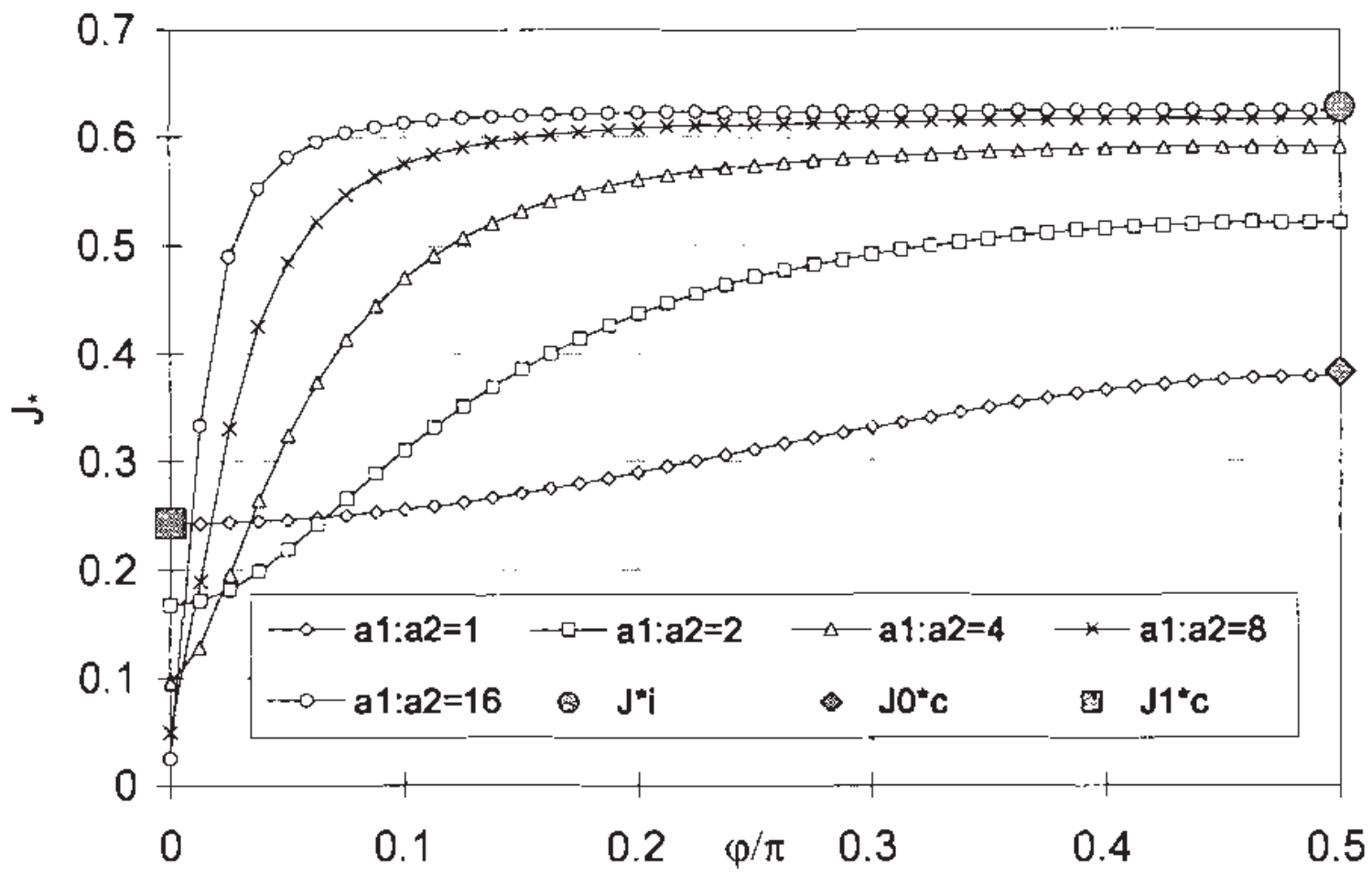

Figure 4 Shear load in the dircction of smaller semiaxis. Dimensionless strain energy release rates for $\nu_{1}=0.4, \nu_{2}=0.2, \mu_{1}: \mu_{2}=1: 4$.

and

$$
J_{*_{c}}^{1}=\frac{4 \pi \varepsilon^{2}\left(1+\varepsilon^{2}\right)^{2}\left(g^{2}-d^{2}\right)^{2} \mu_{1}}{\left\{(g-e) \pi \varepsilon\left(1+\varepsilon^{2}\right)+d\right\}^{2}(g+c)},
$$

respectively. It is shown on Figures 1-3, that the extreme values of $J_{* c}$ are very close to the corresponding values obtained by analytical-numerical method.

There is another possibility to compare our results with analytical solution for a penny-shaped crack. For a crack with radius a subjected to uniform load, the 


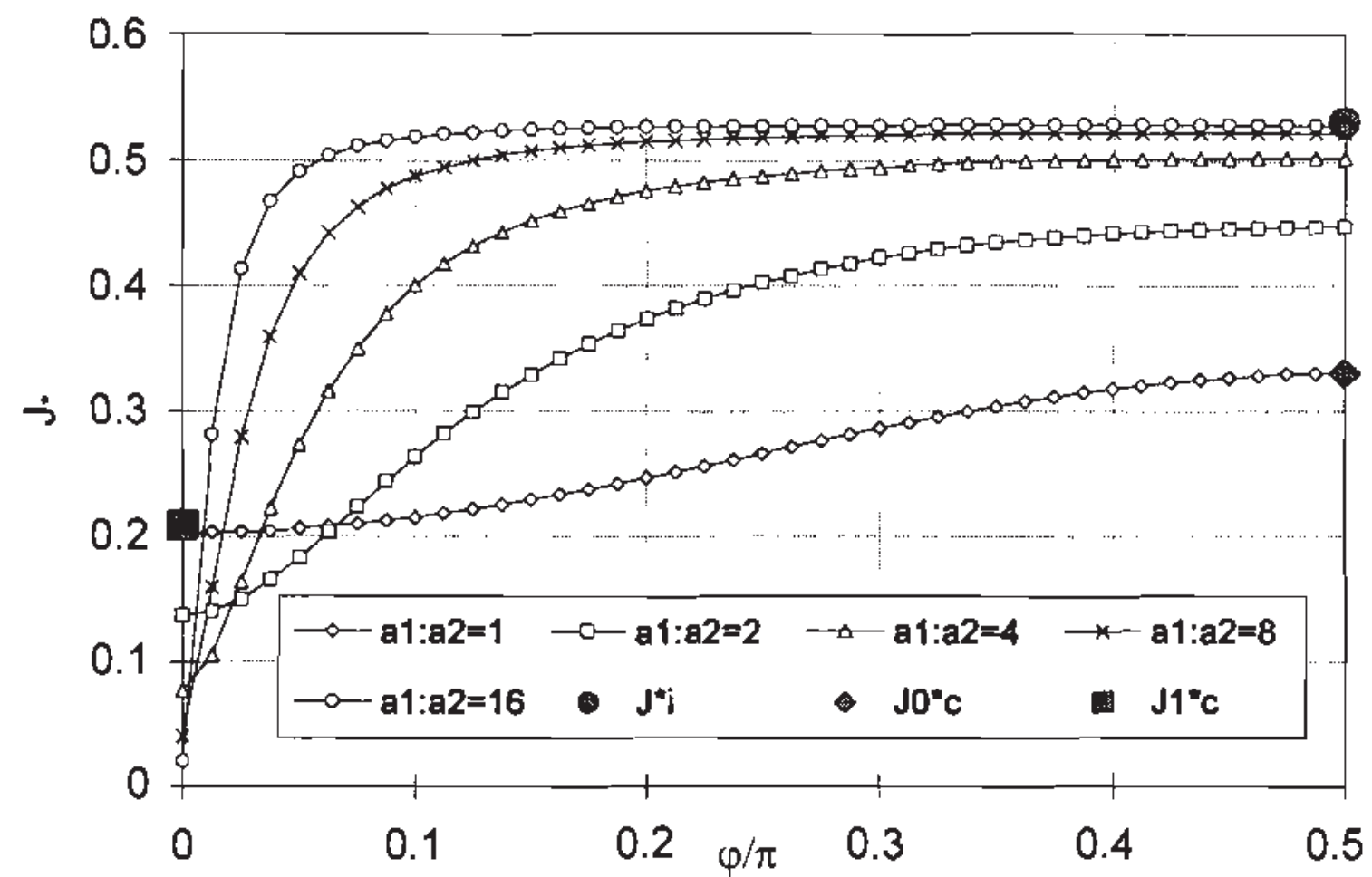

Figure 5 Shear load in the direction of smaller semiaxis. Dimensionless strain energy release rates for $\nu_{1}=0.4, \nu_{2}=0.2, \mu_{1}: \mu_{2}=1: 10$.

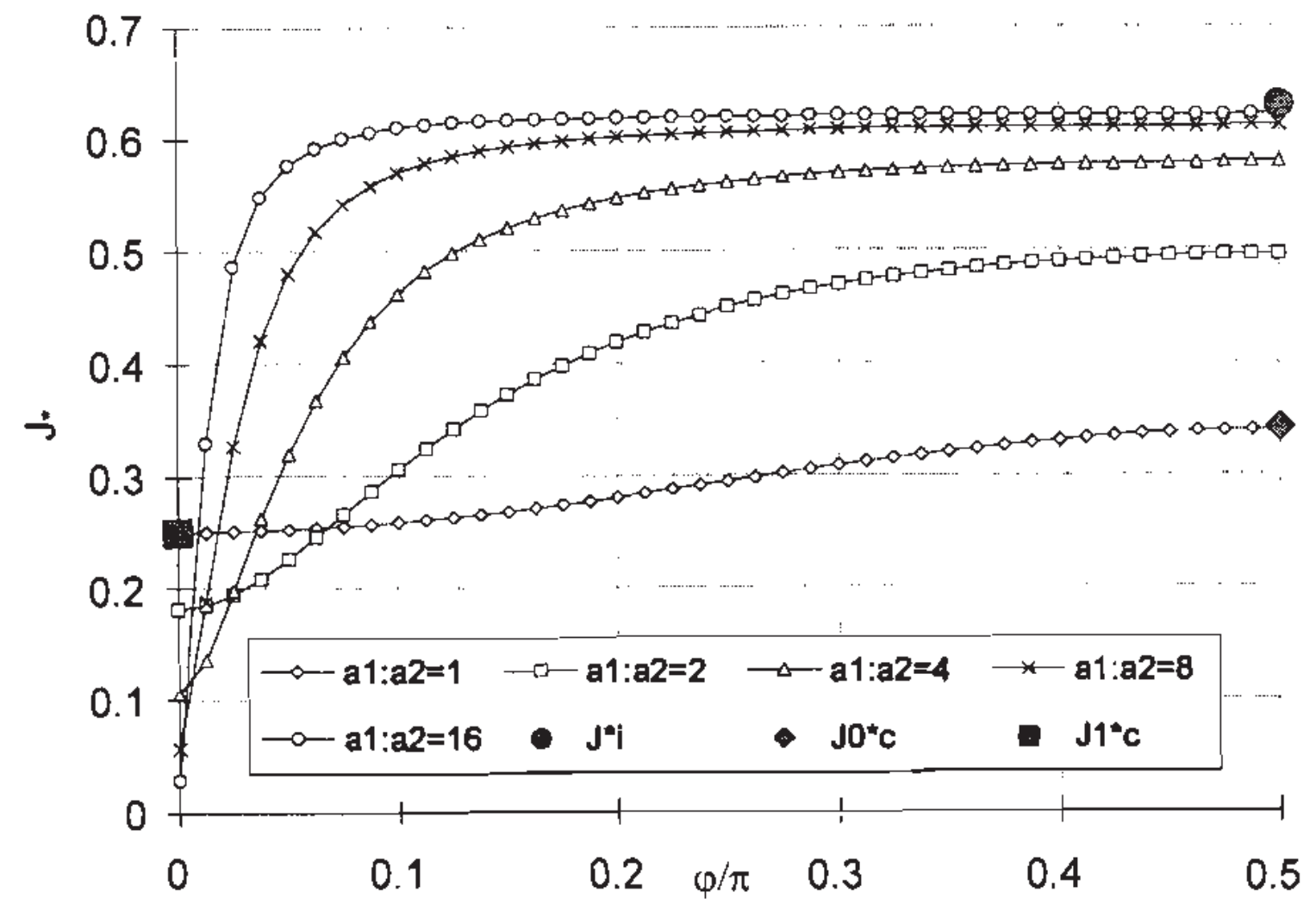

Figure 6 Shear load in the direction of smaller semiaxis. Dimensionless strain energy release rates for $\nu_{1}=0.2, \nu_{2}=0.4, \mu_{1}: \mu_{2}=1: 10$.

following formula is valid

$$
\int_{0}^{2 \pi} J(\varphi) d \varphi=\frac{3 E}{a^{2}},
$$


Table 2 Uniform shear load. The average of the dimensionless strain energy release rates $\overline{J_{*}}$ for a penny-shaped crack.

\begin{tabular}{|c|c|c|c|}
\hline Poisson's ratios & Shear moduli & Formula $(3.5)$ & Analytical-numerical method \\
\hline$\nu_{1}=0.3, \nu_{2}=0.3$ & $\mu_{1}: \mu_{2}=1: 4$ & 0.3244 & 0.3243 \\
\hline$\nu_{1}=0.3, \nu_{2}=0.3$ & $\mu_{1}: \mu_{2}=1: 10$ & 0.2830 & 0.2827 \\
\hline$\nu_{1}=0.2, \nu_{2}=0.4$ & $\mu_{1}: \mu_{2}=1: 4$ & 0.3340 & 0.3334 \\
\hline$\nu_{1}=\overline{0.2, \nu_{2}=0.4}$ & $\mu_{1}: \mu_{2}=1: 10$ & 0.2955 & 0.2946 \\
\hline$\nu_{1}=0.4, \nu_{2}=0.2$ & $\mu_{1}: \mu_{2}=1: 4$ & 0.3104 & $0.31 \overline{04}$ \\
\hline$\nu_{1}=0.4, \nu_{2}=0.2$ & $\mu_{1}: \mu_{2}=1: 10$ & 0.2665 & 0.2665 \\
\hline
\end{tabular}

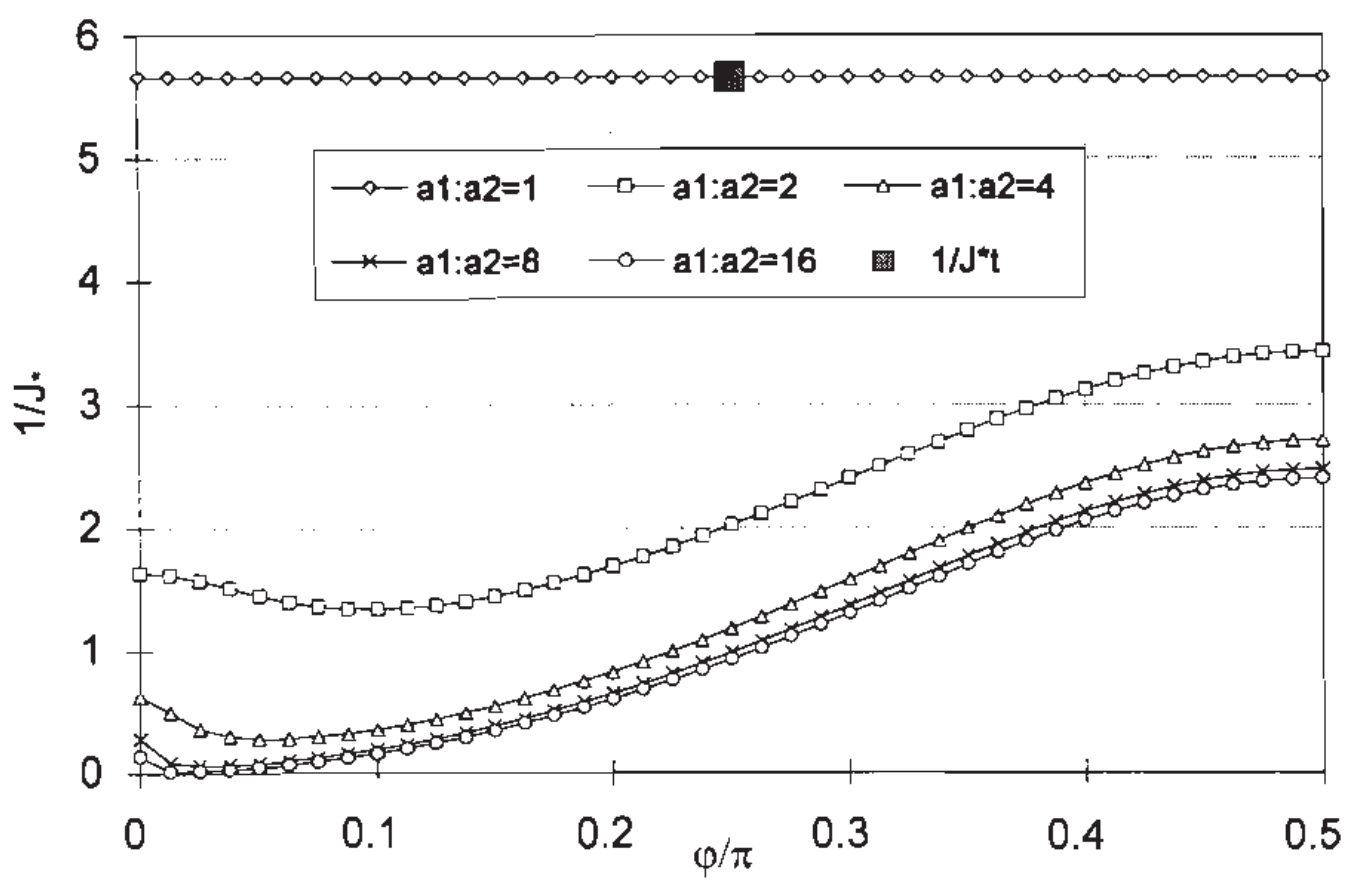

Figure 7 Torsional load. Dimensionless strain energy release rates for $\nu_{1}=$ $\nu_{2}=0.3, \mu_{1}: \mu_{2}=1: 4$.

where $E$ is the total strain energy. Let us now introduce an average of the dimensionless strain energy release rates along the crack contour as

$$
\overline{J_{*}}=\frac{1}{2 \pi} \int_{0}^{2 \pi} J_{*}(\varphi) d \varphi .
$$

From (3.3), (3.4) and the analytical expression for $E$, given in [11], one obtains

$$
\overline{J_{*}}=\frac{2 \varepsilon\left(1+\varepsilon^{2}\right)\left(g^{2}-d^{2}\right) \mu_{1}}{(g-e) \pi \varepsilon\left(1+\varepsilon^{2}\right)+d}
$$

Comparison of $\overline{J_{*}}$ values obtained by analytical formula (3.5) and by numerical integration of function $J_{*}(\varphi)$ within the analytical-numerical method is presented in Table 2. One can see good agreement between exact and numerically obtained results.

3.2 Shear load in the direction of smaller semiaxis. Consider now a uniform shear load acting in the direction of smaller semiaxis

$$
t(x)=(0, \tau, 0)
$$




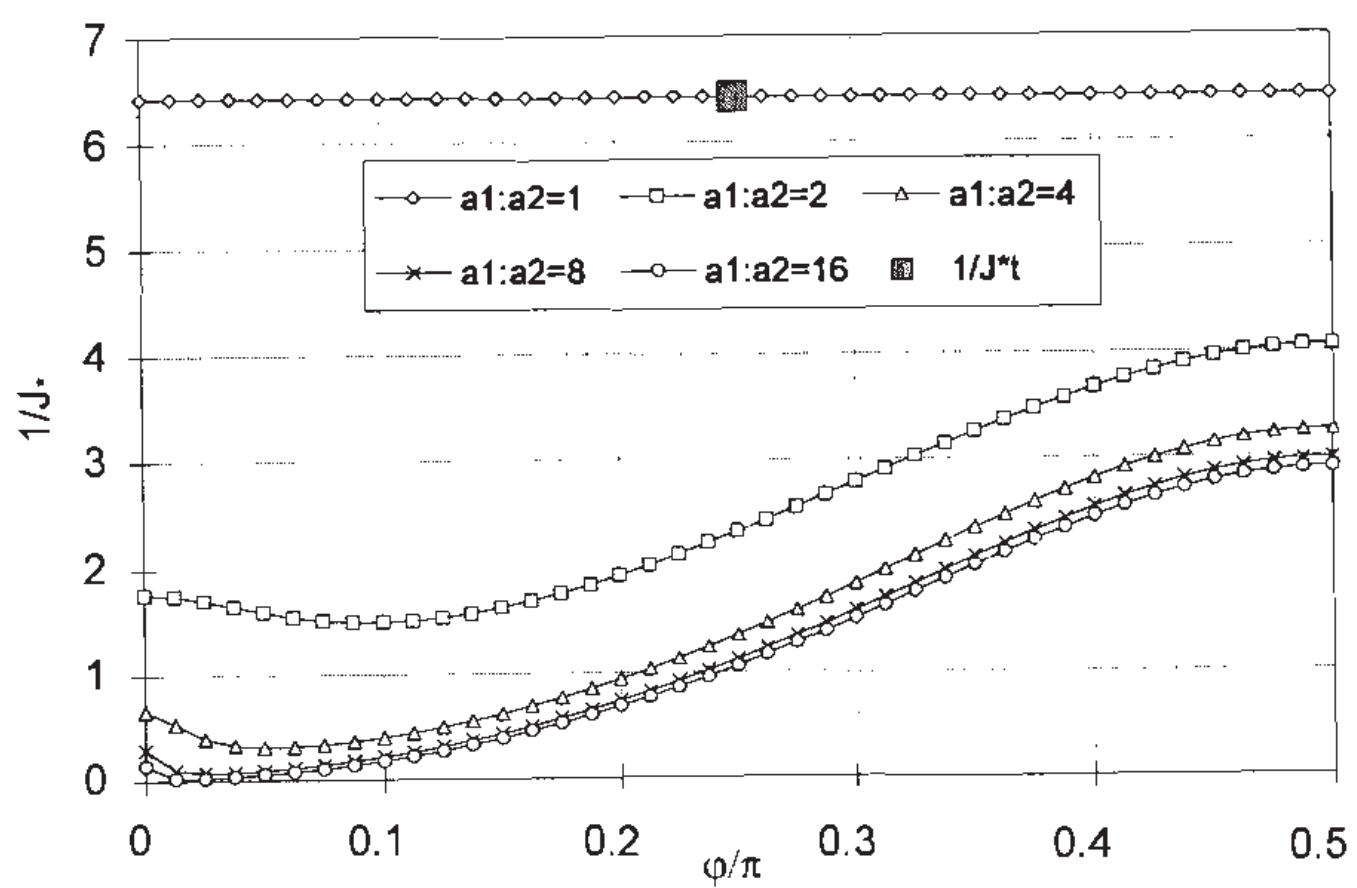

Figure 8 Torsional load. Dimensionless strain energy release rates for $\nu_{1}=$ $0.2, \nu_{2}=0.4, \mu_{1}: \mu^{\prime 2}=1: 10$.

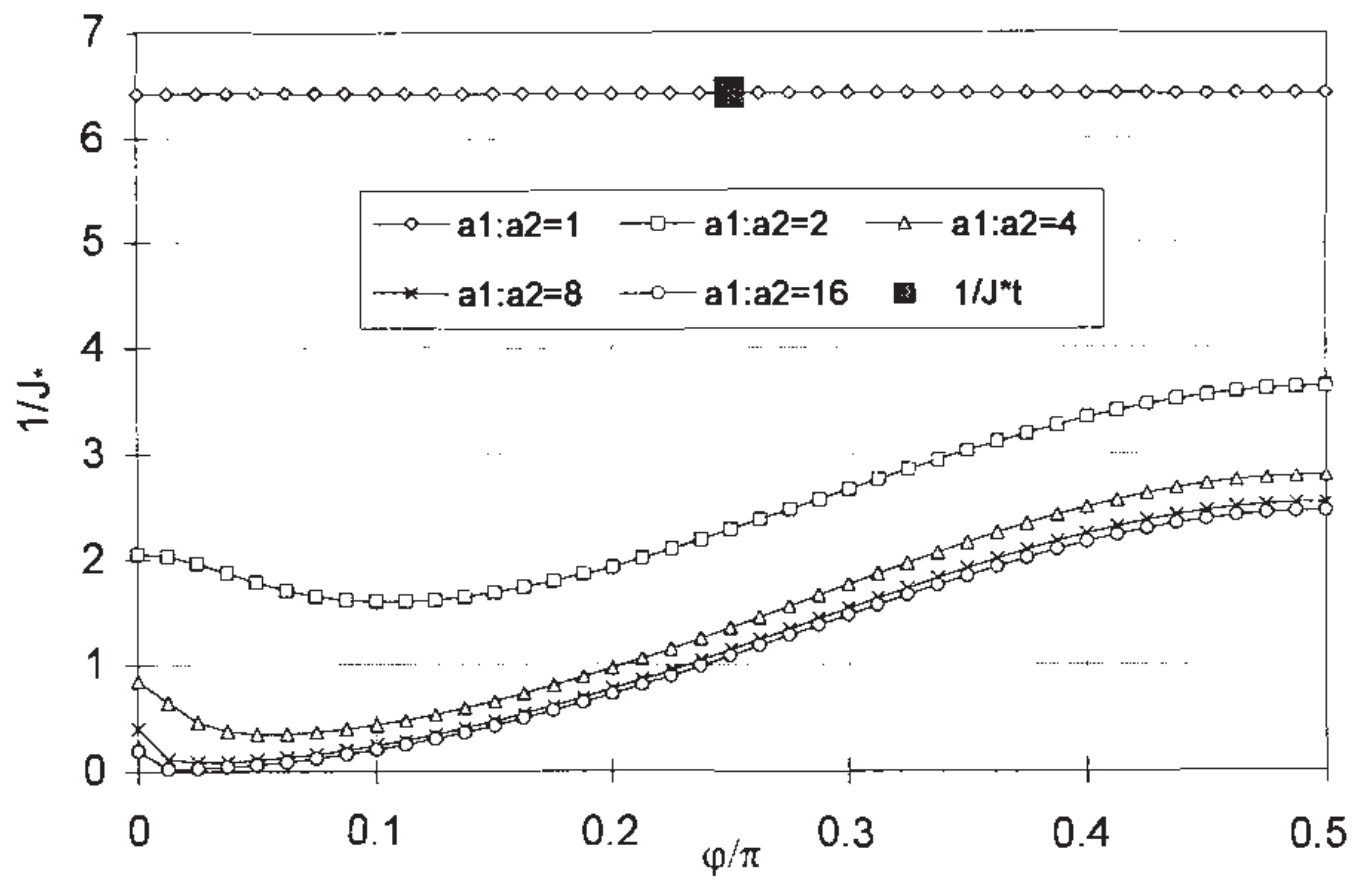

Figure 9 Torsional load. Dimensionless strain energy release rates for $\nu_{1}=$ $0.4, \nu_{2}=0.2, \mu_{1}: \mu_{2}=1: 10$.

Graphs of functions $J_{*}(\varphi)$, where $J_{*}(\varphi)$ is defined according to $(3.1)$, are presented on Figures 4-6.

For this type of load, the value $J_{*}(\pi / 2)$ obtained for elongated elliptical crack have to be close to the value of strain energy release rates $J_{* 2}$ for the interface crack 
of inplane shear with the length $2 a_{2}$. According to [5]

$$
J_{* 2}=\frac{\pi \mu_{1}\left(g^{2}-d^{2}\right)}{4 g}\left(1+4 \varepsilon^{2}\right) .
$$

One can see from Figures $4-6$ that $J_{*}(\pi / 2)$ for elliptical crack with $a_{1}: a_{2}=16$ is only slightly lower than $J_{* i}$.

For a circular crack, the extreme and the average value of the strain energy release rate do not depend on a direction of the load and can be calculated by the formulae presented above. The values of $J_{* c}^{0}$ and $J_{* c}^{\text {l }}$ are indicated on Figures 4-6.

3.3 Torsional load. Finally, let us consider a torsional load of the form

$$
t(x)=\left(-\frac{\tau}{a_{2}} x_{2}, \frac{\tau}{a_{2}} x_{1}, 0\right)
$$

as an example of nonhomogeneous load.

For this loading case, the range of the values of function $J_{*}(\varphi)$ is beconing very large when the crack has a shape of elongated ellipse. It is therefore difficult to present results for different semiaxis ratios on the same figure. For this reason, we rather present dependence of $1 / J_{*}(\varphi)$ with respect to $\varphi$ on an interval $0 \leq \psi \leq \pi / 2$ on Figures 7-9.

For the case of torsional load, the analytical results are known only for the penny-shaped crack. According to [8], the dimensionless values of strain energy release rates $J_{* t}$ are equal to

$$
J_{* t}=\frac{8 \mu_{1}}{9 \pi \mu_{*}} .
$$

Since $J_{* t}$ do not depend on the angle $\varphi$, it is shown only at one point on Figures 7-9. The values $J_{* t}$ exactly coincide with the results obtained by the analyticalnumerical method.

\section{References}

[1] Kaptsov, A.V. and Shifrin, E.I. [1995] Analytical solution of the problem for elliptical crack in elastic medium. I. Statical, normal, polynomial load. Preprint $N^{\prime} 554$. Institute for Problems in Mechanics, Russian Academy of Sciences. Moscow (in Russian).

[2] Kaptsov, A.V. and Shifrin, E.I. [1996] Axalytical solution of the problem for elliptical crack in elastic medium. II. Statical, shear, polynomial load. Preprint $N^{\circ} 559$. Institute for Problems in Mechanics, Russian Acarlemy of Sciences. Moscow (in Russian).

[3] Kassir, M.K. and Bregman, A.M. [1972] The stress-intensity factor for a penny-shaped crack betwcen two dissimilar materials. Journal of Applied Mechanıcs. 39, 308-310.

[4] Mossakovskii, V.I. and Rybka, M.T. [1964] Generalization of the Griffith-Sneddon criterion for the case of a nonhomogeneous body. Joumal of Applaed Mathematios and Mechanzcs [Translation of Prklodnaya Matematrka i Mekhanika (PMM)]. 28, 1277-1286 [1061-1069, Russian edition!.

[5] Salganik R.L. [1963] The brittle fracture of cemented bodies. Journal of Applied Mathematzcs and Mechanics /Translation of Prikladnaya Matematika ? Mekhamzka (PMM)]. 27, 1468-1478 [957-962, Russian edition].

[6] Schwarzer, N., Richter, F. and Michel, B. [1998] Penny shaped interface crack under uniform pressure and shear loading. In: Modeling and stmulation based engineering. Volumc II, Edators: S.N.Atlur, P.E.Donoghue, Published by Tech Sctence Press, USA, 1580-1585.

[7] Shifrin, E.I., Brank, B. ant Surace, G. [1998] Analytical-numerical solution of elliptical interface crack problem. International Journal of Fracture. 94, 201-215.

[8] Sih, G.C. and Chen, E.P. [1981] Mechanics of fractnre. Cracks in composite materials. Volumc 6. Martinus Nighoff Publishers.

[9] Wang, T.-C., Shih, C. F. and Suo., Z. [1992] Crack extension and kinking in laminates and bicristals. International Journal of Solnds ond Structures. 29, 327-344. 
[10] Willis, J.R. [1971] Fracture mechanics of interfacial cracks. Joumal of the Mechanics and Physics of Solids. 19, 353-368.

[11] Willis, J.R. [1972] The penny-shaped crack on an interface. Quarterly Joumal of Mechanics and Appled Mathematics. 25, 367-385. 